\title{
VORWORT ZUR DEUTSCHEN AUSGABE
}

Die bisher in deutscher Sprache erschienenen Lehrbücher und Monographien über Probleme der digitalen Informationsverarbeitung kann man im wesentlichen in zwei Gruppen einteilen.

$\mathrm{Zu}$ der einen Gruppe können die mehr oder weniger stark praktisch orientierten Werke gezählt werden. Sie sind häufig aus Erfahrungen bei der Konstruktion informationsverarbeitender Maschinen oder bei der Nutzung derartiger Anlagen entstanden. Die behandelten Themenstellungen reichen von Fragen der Bauelemententechnik, des Aufbaus einfacher oder komplizierter Schaltungen (etwa von ganzen Rechenwerken) über Probleme der Speichertechnik, der Programmierung (Maschinensprachen, problemorientierte Sprachen, Compiler usw.) und der peripheren Geräte bis zu den Aufgaben, die beim Einsatz von Rechenanlagen und dem Aufbau von Rechenzentren zu lösen sind. Diese Bücher haben vorwiegend beschreibenden Charakter, die mitgeteilten Resultate und vorgeschlagenen Lösungen stammen meistens aus der Erfahrung und sind seltener durch theoretische Überlegungen gewonnen oder gar mathematisch deduziert worden.

In der anderen Gruppe kann man die mehr oder weniger theoretisch orientierten Publikationen zusammenfassen. Sie sind dadurch charakterisiert, daß Schaltungen, Maschinenstrukturen, Automaten und Programmierungsverfahren zum Gegenstand einer mathematischen Betrachtung gemacht und die Resultate durch mathematische Ableitungen gewonnen werden. Diese theoretischen Untersuchungen lassen sich beim heutigen Entwicklungsstand etwa in folgende Einzeldisziplinen aufgliedern: Schaltalgebra, Theorie der abstrakten Automaten, der Kellerautomaten, der TuRING-Maschinen und der stochastischen Automaten, Theorie der Algorithmen und der rekursiven Funktionen, Theorie algorithmischer Sprachen, Codierungs- und Informationstheorie. Die dabei verwendeten mathematischen Methoden entstammen im wesentlichen der Mathematischen Logik, der Algebra, der Wahrscheinlichkeitsrechnung und der Mathematischen Statistik; analytische Betrachtungen dagegen spielen eine untergeordnete Rolle.

Es ist eine Erfahrungstatsache, daß die Abhandlungen der zweiten Gruppe für den Praktiker sehr nützlich sind, ihn jedoch häufig noch schwer ansprechen bzw. ihm wegen ihres hohen Abstraktionsgrades und wegen der nicht unmittelbar sichtbaren technischen und praktischen Bezüge schwer zugänglich sind. Umgekehrt kann man feststellen, daß vorwiegend theoretisch Interessierte die Ab- 
handlungen der ersten Gruppe ungern benutzen, weil sie dort die aus der Mathematik gewohnten korrekten Begriffsbildungen und allgemeinen Methoden vermissen. Es ist deshalb außerordentlich wichtig, hier eine Brücke zu schlagen.

Man muß daher sehr anerkennen, daß sich der Akademie-Verlag Berlin entschlossen hat, das Werk von Kobrinski/Trachtenbrot „Einführung in die Theorie endlicher Automaten" in deutscher Ubersetzung herauszugeben. Dieses Buch zeichnet sich dadurch aus, daß es den Autoren gelungen ist, auf einem wohlabgegrenzten Gebiet (Analyse und Synthese von kombinatorischen und sequentiellen Schaltungen, Beschreibungsverfahren für sequentielle informationsverarbeitende Prozesse) eine enge Verbindung zwischen Theorie und Praxis herzustellen. Für dieses Vorhaben sind die Autoren ideal prädestiniert; KoBRINSKI verfügt über große praktische Erfahrungen auf dem Gebiet der Elektronik und Rechentechnik, Trachtenвrot dagegen beherrscht als Logiker und Mathematiker hervorragend die theoretischen Methoden. Das hier vorliegende Buch wendet sich gleichermaßen an den Mathematiker wie an den Techniker. Dem Praktiker wird das Verständnis dadurch erleichtert, daß die z.T. tiefliegenden theoretischen Resultate durch viele Beispiele erläutert werden und zugunsten einer möglichst verständlichen Darstellung die mathematischen Betrachtungen und die Beweise häufig nicht in voller Allgemeinheit durchgeführt werden. Der Mathematiker dagegen sollte das nicht als Mangel empfinden; ihm dürfte es zum Beispiel nicht schwerfallen, sich (evtl. anhand von zusätzlicher Literatur) die volle Allgemeinheit der Darlegungen selbst herzustellen. Für ihn hat die Lektüre dieses Buches vielmehr den großen Vorteil, daß er dadurch angehalten wird, seine mathematischen Kenntnisse mit technischen Vorstellungen zu verbinden.

Das vorliegende Werk wurde im Jahre 1958 begonnen und 1962 herausgegeben. Man muß deshalb die Frage stellen, ob es angesichts der stürmischen Entwicklung der Kybernetik inzwischen veraltet ist und eine Herausgabe in deutscher Sprache sich nicht mehr lohnt. Diese Frage muß entschieden verneint werden, weil die meisten der in dem Buch enthaltenen Resultate eine tiefliegende und prinzipielle Bedeutung haben und von bleibendem Wert sind. Daran ändert auch die Tatsache nichts, daß die technische Entwicklung in den letzten zehn Jahren sehr schnell vorangegangen ist und demgemäß die in Kapitel III enthaltenen Betrachtungen über Bauelemente und Baugruppen sowie einige Beispiele aus Kapitel IV heute zum Teil als überholt angesehen werden müssen.

Die Herausgeber der deutschen Ausgabe haben an einigen Stellen, die hier nicht im einzelnen aufgezählt werden können, im Text aber meistens gekennzeichnet sind, etwas stärker, als es sonst bei Ubersetzungen üblich ist, in die Struktur des Originals eingegriffen. Zunächst ist zu sagen, daß unter Verzicht auf eine Satz-für-Satz-Ưbertragung (oder gar eine wörtliche Ưbersetzung) eine dem heutigen Entwicklungsstand möglichst entsprechende Darstellung angestrebt worden ist. Dazu wurden häufig Definitionen und Erläuterungen, die im Original fehlten oder nur kurz angedeutet waren, hinzugefügt bzw. vervollstän- 
digt. Es ist ferner selbstverständlich, daß eine Reihe kleinerer und größerer sachlicher Unkorrektheiten und Fehler ausgemerzt worden ist. Ein stärkerer Eingriff in das Original besteht darin, daß der Begriff des abstrakten Automaten, wie er dem deutschen Leser insbesondere durch die Übersetzung W. M. GLuschkow ,Theorie der abstrakten Automaten“" (VEB Deutscher Verlag der Wissenschaften, Berlin 1963) bekanntgeworden ist, in die Ubersetzung aufgenommen und konsequent verwendet wird. Darüber hinaus wurde, um das Wichtigste über abstrakte Automaten kurz zusammengefaßt darstellen zu können, in Kapitel II ein neuer Paragraph (§ 3) eingefügt. Die Herausgeber der deutschen Ausgabe fühlten sich gegenüber Autoren und Lesern aus folgenden Gründen zu diesen Abänderungen verpflichtet und berechtigt. Zur Zeit der Abfassung des Werkes hatte sich die Theorie der abstrakten Automaten eben erst über die Anfänge hinaus entwickelt, und der Begriff des abstrakten Automaten war im Grunde genommen noch nicht voll präzisiert. Dies ist die Ursache dafür, daß im Original die Beziehungen zwischen Automatenoperatoren und den sogenannten kanonischen Gleichungen für Automatenoperatoren (die kanonischen Gleichungen charakterisieren, modern ausgedrückt, die Arbeitsweise eines abstrakten Automaten, in dem der betrachtete Automatenoperator dargestellt wird) nicht völlig klar sind. Es kann angenommen werden, daß diese Abänderungen und Ergänzungen die Lektüre der vorliegenden Übersetzung erleichtern. Durch ein zusätzliches Verzeichnis einschlägiger Arbeiten, die nach Erscheinen des Originals publiziert wurden, soll der Leser auf einige Aspekte der weiteren Entwicklung hingewiesen werden.

Es wurde darauf verzichtet, im Kapitel III über das Original hinausgehend Bauelemente, die inzwischen in den Mittelpunkt des Interesses gerückt sind, zu besprechen. Ein Hinzufügen derartiger Beschreibungen allein hätte der Gesamtkonzeption dieses Buches widersprochen; eine enge Verknüpfung mit den späteren theoretischen Betrachtungen und Beispielen wäre dagegen mit einem zu starken Eingriff in den Gesamtaufbau verbunden gewesen.

Den Herausgebern der deutschen Ausgabe bleibt zum Schluß die angenehme Pflicht, Dank zu sagen; an erster Stelle den beiden Autoren für ihr Verständnis hinsichtlich der angedeuteten Übersetzungsprobleme und für ihre Hilfe bei der Beseitigung von Fehlern im Original. Dem Akademie-Verlag, Berlin, sei gedankt für die Initiative zur deutschen Herausgabe dieses Werkes und für das verständnisvolle Eingehen auf unsere Wünsche bei Drucklegung und Korrektur; in gleicher Weise gebührt auch Dank dem VEB Druckerei ,Thomas Müntzer“, Bad Langensalza, für die gute Arbeit bei der Gesamtherstellung.

Schließlich möchten wir herzlich danken einigen Studenten der Sektion Mathematik der Friedrich-Schiller-Universität Jena für die Hilfe bei den Korrekturen, der Anfertigung der Register und der Zusammenstellung des zusätzlichen Literaturverzeichnisses, insbesondere Herrn K. Fischer, jedoch auch den Herren L. Bernhardt, H.-G. KlepPe, G. Kummer.

Jena, im Mai 1967

Die Herausgeber der deutschen Ausgabe 